\title{
Effects of Quark Interactions on Dynamical Chiral Symmetry Breaking by a Magnetic Field ${ }^{\star}$
}

\author{
Brigitte HILLER ${ }^{\dagger}$, Alexander A. OSIPOV ${ }^{\ddagger}$, Alex H. BLIN ${ }^{\dagger}$ and João da PROVIDÊNCIA ${ }^{\dagger}$ \\ † Centro de Física Teórica, Departamento de Física da Universidade de Coimbra, \\ 3004-516 Coimbra, Portugal \\ E-mail: brigitte@teor.fis.uc.pt, alex@teor.fis.uc.pt, providencia@teor.fis.uc.pt \\ $\ddagger$ Joint Institute for Nuclear Research, Laboratory of Nuclear Problems, \\ 141980 Dubna, Moscow region, Russia \\ E-mail: osipov@nu.jinr.ru
}

Received November 14, 2007, in final form February 07, 2008; Published online February 22, 2008

Original article is available at http://www.emis.de/journals/SIGMA/2008/024/

\begin{abstract}
It is shown how the strong interaction dynamics of a multi-quark Lagrangian affects the catalysis of dynamical symmetry breaking by a constant magnetic field in $(3+1)$ dimensions. Attention is drawn to the local minima structure of the theory.
\end{abstract}

Key words: dynamical chiral symmetry breaking; catalysis; field theoretical model of multiquark interactions

2000 Mathematics Subject Classification: $81 \mathrm{~T} 10$

\section{Introduction}

The existence of a zero-energy surface in the spectrum of a Dirac particle is ensured for any homogeneous magnetic field with a fixed direction by a quantum mechanical supersymmetry of the corresponding second-order Dirac Hamiltonian [1]. For interacting fermions subject to an attractive two-body force in $2+1$ and $3+1$ dimensions, a constant magnetic field catalyzes the dynamical symmetry breaking leading to fermion pairing and to a fermion mass even for arbitrarily weak coupling strength $[2,3,4]$. The zero-energy surface of the lowest Landau level (LLL) plays a crucial role in the dynamics of such fermion pairing $[5,6,7]$, and the generated fermion mass, $M_{\text {dyn }}$, turns out to be much smaller than the Landau gap $\sim \sqrt{|e H|}$. The dynamics of the fermion pairing in the homogeneous magnetic field is essentially $(1+1)$-dimensional, and the deep analogy of this phenomenon with the dynamics of electron pairing in BCS [8] has been stressed. The generation of mass via catalysis does however not prevail for arbitrary magnetic field configurations. For instance, it has been demonstrated in $[9,10]$ that the NambuJona-Lasinio (NJL) model [11] minimally coupled to a background magnetic field with variable direction becomes massive only beyond some critical value of the strong coupling constant. In these cases dynamical chiral symmetry breaking corresponds to the standard scenario.

In the present paper we show and discuss the influence that the structure of interaction terms, specified below, may have on the effect of catalysis by a constant magnetic field (from now on we use interaction terms to denote interaction among the fermions, the interaction with the magnetic field will be explicitly referred as such). We obtain that catalysis of dynamical symmetry breaking always occurs at arbitrarily small magnetic fields for a system which is subcritical in the interaction couplings in absence of the magnetic field. However for increasing

\footnotetext{
${ }^{\star}$ This paper is a contribution to the Proceedings of the Seventh International Conference "Symmetry in Nonlinear Mathematical Physics" (June 24-30, 2007, Kyiv, Ukraine). The full collection is available at http://www.emis.de/journals/SIGMA/symmetry2007.html
} 
strength of the magnetic field and depending on the values of interaction couplings, another minimum of the effective potential emerges at larger dynamical fermion masses, which competes with the catalyzed minimum and eventually becomes the globally stable state of the system at characteristic scales $H=10^{19}$ Gauss. The broken symmetry is not restored with increasing strength of the magnetic field.

A few words about the fermion interaction model used in this paper: in recent years we have reported on the importance of adding eight-quark interaction terms [12] to the four-quark $U(3)_{L} \times U(3)_{R}$ chiral symmetric NJL Lagrangian joined with the $U(1)_{A}$ breaking 't Hooft sixquark interaction [13]. Their inclusion leads to a scalar effective potential which is globally stable and to a full equivalence between the stationary phase approach (SPA) and the mean field (MF) approximation methods used to obtain it. Without them the model is afflicted by an unstable (SPA) respectively metastable (MF) vacuum [14], as presented two years ago at the Kyiv 2005 conference [15]. There we have also shown that going beyond the leading order results of SPA did not cure the instability of the effective potential. Although we prefer to consider the inclusion of eight quark interactions as the minimal chiral approach to render the vacuum of the NJL model with 't Hooft interaction stable, QCD regarded as an effective theory displays an infinite chain of multiquark interactions [16]. The issue of the corresponding $N_{c}$ counting rules is however still open. In the framework of the instanton gas model beyond the zero mode approximation multiquark interactions are delivered at any $2 n$-quark order $(n \geq 2)$ with equal weight in the large $N_{c}$ limit [17]. On the other hand lattice calculations of gluon correlators [18] give a larger weight to the lower correlators. This leads to the expectation that after integrating out the gluonic degrees of freedom, a similar hierarchy could arise for the multiquark interactions. In our approach higher order interactions are assumed to be suppressed in the large $N_{c}$ counting $[12,19]$.

Since then the eight-quark extended model has been carefully studied in connection with the low lying spectra and characteristics of the pseudoscalar and scalar nonets [19], temperature dependence of chiral transitions [20], and [21] for the two flavor case, and coupling to a constant magnetic field [22]. We have learned that the effects of the eight quark interactions on the mass spectra are not essential, except for the mass of the lowest state of the mixed singlet-octet scalar states, which decreases with increasing strength of the eight quark interaction terms that violate the Okubo-Zweig-Iizuka (OZI) rule [23]. However, their effect can be dramatic on studies involving changes of the extrema of the effective potential, which are for instance the cases related with the thermodynamics, the in medium dependence, and the coupling to electromagnetic fields of the multi-fermion system. The latter is the main subject of the present contribution. This work is very much based on our recent paper [22], it is however a more extensive version. It contains also truly novel calculations, as we chose as starting configurations a regime of completely sub-critical solutions (i.e. only the trivial vacuum with vanishing condensate exists in absence of the magnetic field), which were not analyzed before for the enlarged multi-quark Lagrangian. We study then how the system responds to coupling to a magnetic field, starting with an arbitrarily weak strength and increasing it successively. First appear the well known catalyzed solutions as minima of the gap equation. Then the fate of the system may be either a first order transition or a crossover to heavier condensates, depending on the strength of the couplings of multiquark interactions. The paper is structured as follows. In Section 2 we present the model Lagrangian in presence of a constant magnetic field and the corresponding effective potential. In Section 3 we obtain the solutions of the gap equations and their relation with the Landau levels and highlight the differences to the well-known cases. In Section 4 we present the conclusions. 


\section{Model Lagrangian}

In the hadronic QCD vacuum the relevant fermionic interactions can be modeled by the wellknown Nambu-Jona-Lasinio (NJL) Lagrangian [11]. The coupling to a constant magnetic field has been done in [6] for one-flavour fermions.

For a more realistic analysis one should take into account the different quark flavours of the QCD vacuum. These extensions of the NJL model are well-known [24, 25, 26], for instance, the four-quark $U(3)_{L} \times U(3)_{R}$ chiral symmetric Lagrangian together with the $U(1)_{A}$ breaking 't Hooft six-quark interactions has been extensively studied at the mean-field level [27, 28, 29, 30, 31]. As mentioned in the introduction, it has been recently shown [12] that the eight-quark interactions are of vital importance to stabilize the multi-quark vacuum.

The multi-quark dynamics of the extended NJL model is described by the Lagrangian density

$$
\mathcal{L}_{\text {eff }}=\bar{q}\left(i \gamma^{\mu} D_{\mu}-\hat{m}\right) q+\mathcal{L}_{4 q}+\mathcal{L}_{6 q}+\mathcal{L}_{8 q},
$$

where the gauge covariant derivative $D_{\mu}$ is equal to $D_{\mu}=\partial_{\mu}+i Q A_{\mu}$ with the external electromagnetic field $A_{\mu}$ and quark charges $Q=e \cdot \operatorname{diag}(2 / 3,-1 / 3,-1 / 3)$. We consider a constant magnetic field, $A_{x}=-H y, A_{y}=A_{z}=0$ (Landau gauge). It is assumed that quark fields have colour $\left(N_{c}=3\right)$ and flavour $\left(N_{f}=3\right)$ indices. The current quark mass, $\hat{m}$, is a diagonal matrix with elements $\operatorname{diag}\left(\hat{m}_{u}, \hat{m}_{d}, \hat{m}_{s}\right)$, which explicitly breaks the global chiral $S U_{L}(3) \times S U_{R}(3)$ symmetry of the Lagrangian. We shall neglect this effect in the following assuming that $\hat{m}=0$.

The most general form of multi-quark interactions in the scalar and pseudoscalar channels are of the form

$$
\mathcal{L}_{4 q}=\frac{G}{2}\left[\left(\bar{q} \lambda_{a} q\right)^{2}+\left(\bar{q} i \gamma_{5} \lambda_{a} q\right)^{2}\right], \quad \mathcal{L}_{6 q}=\kappa\left(\operatorname{det} \bar{q} P_{L} q+\operatorname{det} \bar{q} P_{R} q\right), \quad \mathcal{L}_{8 q}=\mathcal{L}_{8 q}^{(1)}+\mathcal{L}_{8 q}^{(2)} .
$$

The $U(3)$ flavour matrices $\lambda_{a}, a=0,1, \ldots, 8$, are normalized such that $\operatorname{tr}\left(\lambda_{a} \lambda_{b}\right)=2 \delta_{a b}$. The matrices $P_{L, R}=\left(1 \mp \gamma_{5}\right) / 2$ are chiral projectors and the determinant is over flavour indices, which are suppressed here. The determinantal interaction breaks explicitly the axial $U(1)_{A}$ symmetry [13] and Zweig's rule. The eight-quark spin zero interactions are given by

$$
\mathcal{L}_{8 q}^{(1)}=8 g_{1}\left[\left(\bar{q}_{i} P_{R} q_{m}\right)\left(\bar{q}_{m} P_{L} q_{i}\right)\right]^{2}, \quad \mathcal{L}_{8 q}^{(2)}=16 g_{2}\left(\bar{q}_{i} P_{R} q_{m}\right)\left(\bar{q}_{m} P_{L} q_{j}\right)\left(\bar{q}_{j} P_{R} q_{k}\right)\left(\bar{q}_{k} P_{L} q_{i}\right) .
$$

$G, \kappa, g_{1}, g_{2}$ are dimensionful coupling constants: $[G]=M^{-2},[\kappa]=M^{-5},\left[g_{1}\right]=\left[g_{2}\right]=M^{-8}$ in units $\hbar=c=1$. The term proportional to $g_{1}$ violates the OZI rule.

\section{$2.1 \quad$ Effective potential}

We proceed by calculating the effective potential of the theory, $V\left(m_{u}, m_{d}, m_{s}\right)$, in the constant magnetic field $A_{x}=-H y, A_{y}=A_{z}=0$. The arguments, $m_{i}$, are simply real parameters; they are not to be identified with the masses of any presumed one-particle states. Instead, we shall use the capital letter $M_{i}$ for the point where $V$ takes its local minimum, which specifies the masses of constituent quark fields.

The potential is built of the following two terms

$$
V\left(m_{u}, m_{d}, m_{s}\right)=V_{\text {st }}+V_{S} .
$$

The first contribution results from the many-fermion vertices of Lagrangian $\mathcal{L}_{\text {eff }}$, after reducing them to a bilinear form with help of bosonic auxiliary fields, and subsequent integration over these fields, using the stationary phase method. The specific details of these calculations and the result are given in [12]. We obtain

$$
V_{\mathrm{st}}=\frac{1}{16}\left(4 G h_{i}^{2}+\kappa h_{u} h_{d} h_{s}+\frac{3 g_{1}}{2}\left(h_{i}^{2}\right)^{2}+3 g_{2} h_{i}^{4}\right)
$$


where $h_{i}^{2}=h_{u}^{2}+h_{d}^{2}+h_{s}^{2}$, and $h_{i}^{4}=h_{u}^{4}+h_{d}^{4}+h_{s}^{4}$. The functions $h_{i}$ depend on the coupling constants $G, \kappa, g_{1}, g_{2}$ and on the independent variables $\Delta_{i}=m_{i}-\hat{m}_{i}$. To find this dependence one should solve the system of cubic equations

$$
\begin{aligned}
G h_{u}+\Delta_{u}+\frac{\kappa}{16} h_{d} h_{s}+\frac{g_{1}}{4} h_{u} h_{i}^{2}+\frac{g_{2}}{2} h_{u}^{3}=0, \\
G h_{d}+\Delta_{d}+\frac{\kappa}{16} h_{u} h_{s}+\frac{g_{1}}{4} h_{d} h_{i}^{2}+\frac{g_{2}}{2} h_{d}^{3}=0, \\
G h_{s}+\Delta_{s}+\frac{\kappa}{16} h_{u} h_{d}+\frac{g_{1}}{4} h_{s} h_{i}^{2}+\frac{g_{2}}{2} h_{s}^{3}=0 .
\end{aligned}
$$

In the parameter range

$$
g_{1}>0, \quad g_{1}+3 g_{2}>0, \quad G>\frac{1}{g_{1}}\left(\frac{\kappa}{16}\right)^{2} .
$$

the system has only one set of real solutions, and this guarantees the vacuum state of the theory to be stable [12].

The second term on the r.h.s. of equation (1) derives from the integration over the quark bilinears in the functional integral of the theory in presence of a constant magnetic field $H$. As has been calculated by Schwinger a long time ago [32]

$$
V_{S}=\sum_{i=u, d, s} V_{S}\left(m_{i},\left|Q_{i} H\right|\right)
$$

where

$$
V_{S}(m,|Q H|)=\frac{N_{c}}{8 \pi^{2}} \int_{0}^{\infty} \frac{d s}{s^{2}} e^{-s m^{2}} \rho\left(s, \Lambda^{2}\right)|Q H| \operatorname{coth}(s|Q H|),
$$

up to an unessential constant. Here the cutoff $\Lambda$ has been introduced by subtracting off suitable counterterms to regularize the integral at the lower limit, i.e., $\rho\left(s, \Lambda^{2}\right)=1-\left(1+s \Lambda^{2}\right) e^{-s \Lambda^{2}}$. For the fermion tadpole this works as the four-momentum covariant cutoff in the Euclidean space: $\vec{p}^{2}+p_{4}^{2}<\Lambda^{2}$. As a result we obtain

$$
\begin{aligned}
V_{S}(m,|Q H|)= & \frac{N_{c}}{8 \pi^{2}}\left\{\Lambda^{2}|Q H|\left[\ln 2 \pi-2 \ln \Gamma\left(\frac{\Lambda^{2}+m^{2}}{2|Q H|}\right)\right]+m^{2}|Q H| \ln \left(1+\frac{\Lambda^{2}}{m^{2}}\right)\right. \\
& +\left.4(Q H)^{2} \frac{d}{d \nu}\left[\zeta\left(\nu-1, \frac{\Lambda^{2}+m^{2}}{2|Q H|}\right)-\zeta\left(\nu-1, \frac{m^{2}}{2|Q H|}\right)\right]\right|_{\nu=0} \\
& \left.+\frac{\Lambda^{4}}{2}\left(\ln \frac{\Lambda^{2}}{2|Q H|}-\frac{3}{2}\right)-\Lambda^{2} m^{2}\right\} .
\end{aligned}
$$

The quantity $\zeta(\nu, x)$ denotes the generalized Riemann zeta function [33].

\section{Analysis of solutions}

In this section we study the extrema of the effective potential, given by the gap equations. We start by considering the known case with four-quark interactions in Subsection 3.1. A detailed study of several relevant limits and the role of Landau levels in the catalysis of dynamical symmetry breaking is presented. Then in Subsection 3.2 the extended system with six and eight quark interactions is analyzed and its new features discussed. 


\subsection{The standard four-quark system in a constant magnetic field}

We consider first the simple $S U(3)$ flavour limit for the situation in which $\hat{m}=0$ and $\kappa=$ $g_{1}=g_{2}=0$. For purposes of illustration, we ignore in the remaining the charge difference of $u$ and $d, s$ quarks. The averaged common charge $|Q|=|4 e / 9|$ will be used. In this case one gets the potential $V(m)=N_{f}\left(m^{2} / 4 G+V_{S}(m,|Q H|)\right)^{1}$ and the gap equation is obtained as

$$
\begin{aligned}
\frac{d V(m)}{d m}= & m N_{c} N_{f}\left\{\frac{1}{2 G N_{c}}-\frac{\Lambda^{2}}{4 \pi^{2}} \psi\left(\frac{\Lambda^{2}+m^{2}}{2|Q H|}\right)\right. \\
& \left.+\frac{|Q H|}{4 \pi^{2}}\left[J_{1}\left(m^{2}\right)+2 \ln \frac{\Gamma\left(\frac{\Lambda^{2}+m^{2}}{2|Q H|}\right)}{\Gamma\left(\frac{m^{2}}{2|Q H|}\right)}\right]\right\}=0,
\end{aligned}
$$

where $\psi(x)=d \ln \Gamma(x) / d x$ is the Euler dilogarithmic function, and

$$
J_{1}\left(m^{2}\right)=\ln \left(1+\frac{\Lambda^{2}}{m^{2}}\right)-\frac{\Lambda^{2}}{\Lambda^{2}+m^{2}} .
$$

The gap equation always has a trivial solution, $m=0$. The nontrivial solution is contained in the equation

$$
\frac{2 \pi^{2}}{G \Lambda^{2} N_{c}}=f\left(m^{2} ; \Lambda,|Q H|\right) \equiv \psi\left(\frac{\Lambda^{2}+m^{2}}{2|Q H|}\right)-\frac{|Q H|}{\Lambda^{2}}\left[J_{1}\left(m^{2}\right)+2 \ln \frac{\Gamma\left(\frac{\Lambda^{2}+m^{2}}{2|Q H|}\right)}{\Gamma\left(\frac{m^{2}}{2|Q H|}\right)}\right] .
$$

Note that although our regularization scheme differs essentially from the one used in [6], the main conclusion of that paper is not changed: as in [6], our gap equation has a nontrivial solution at all $G>0$, if $H \neq 0$. Fig. 1 illustrates this important result. Displayed are the r.h.s. (curves $f$ ) and l.h.s. of equation (4), shown only for the positive valued abscissa, since the curves are symmetric. The numerical value of the four-quark coupling is taken to be $G \Lambda^{2}=3$. The l.h.s. is a constant and indicated by the short-dashed line. The r.h.s. is shown as the long-dashed curve (for $H=0$ ) and as full lines for $|Q H| \Lambda^{-2}=0.1$ and $|Q H| \Lambda^{-2}=0.5$. One sees that in absence of the magnetic field one is in the subcritical regime of dynamical symmetry breaking, for this choice of $G \Lambda^{2}$. The inclusion of a constant magnetic field, however small it might be, changes radically the dynamical symmetry breaking pattern, due to the singular behaviour of the r.h.s. close to the origin: the crossing of right and left hand sides of equation (4) does always occur and the value of $m$ where this happens is a minimum of the effective potential. In turn the trivial solution, which at $H=0$ is a minimum, is transformed into a maximum for $H \neq 0$.

Let us look at these different regimes more closely. As $H \rightarrow 0$, we recover the well-known NJL model gap equation

$$
1=\frac{G N_{c}}{2 \pi^{2}}\left(J_{0}\left(m^{2}\right)+\frac{|Q H|^{2} \Lambda^{2}}{3 m^{2}\left(\Lambda^{2}+m^{2}\right)}+\cdots\right),
$$

where $J_{0}\left(m^{2}\right)$ is

$$
J_{0}\left(m^{2}\right)=\Lambda^{2}-m^{2} \ln \left(1+\frac{\Lambda^{2}}{m^{2}}\right) .
$$

Equation (5) at $H=0$ admits a nontrivial solution only if $\tau>1$, where $\tau=G \Lambda^{2} N_{c} / 2 \pi^{2}$. This determines the critical value $G_{\text {crit }}=2 \pi^{2} / \Lambda^{2} N_{c}$. The coupling $G$ is said to be overcritical if $G>G_{\text {crit }}$, and subcritical in the opposite case when $G<G_{\text {crit }}$.

\footnotetext{
${ }^{1}$ Note that the $S U(3)$ result for the effective potential divided by the number of flavours does not coincide with the one flavour case. This is due to group structure factors.
} 


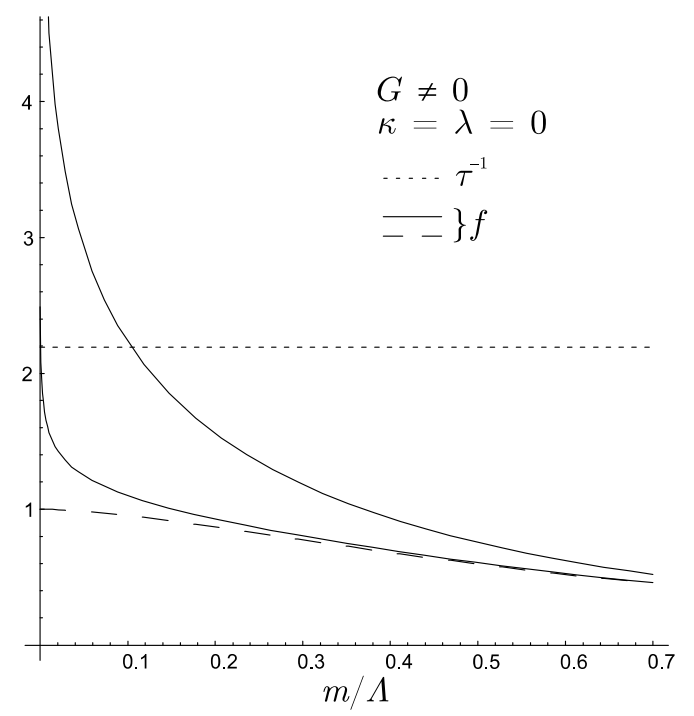

Figure 1. The the 1.h.s. (straight short-dashed line) and the r.h.s. (other curves) of the gap equation (4) related to the four-quark Lagrangian, as functions of $m / \Lambda$, all in dimensionless units. Three different values of the magnetic field strength $H$ are considered: the long-dashed curve corresponds to $H=0$; the two full curves represent the cases for magnetic fields of strength $|Q H| \Lambda^{-2}=0.5$ (upper curve) and $|Q H| \Lambda^{-2}=0.1$. For any $H \neq 0$ the l.h.s. and r.h.s. intersect and yield the non-trivial solution of (4). For $H=0$ the system is subcritical for the parameter set considered. It becomes critical in the case $\tau>1$, where $\tau=G \Lambda^{2} N_{c} / 2 \pi^{2}$.

At $m^{2} / \Lambda^{2} \ll 1$ the r.h.s. of equation (4) is

$$
-\frac{|Q H|}{\Lambda^{2}} \ln \left(\frac{m^{2}}{\Lambda^{2}}\right)+v(\xi)+\mathcal{O}\left(\frac{m^{2}}{\Lambda^{2}}\right) .
$$

Here the function $v(\xi)$ of the argument $\xi=\Lambda^{2} / 2|Q H|$, is given by

$$
v(\xi)=\frac{1}{2 \xi}[1-2 \ln \Gamma(\xi+1)]+\psi(\xi) .
$$

This is a monotonically increasing function on the interval $0<\xi<\infty ; v(\xi)=0$ at the point $\xi \simeq 1.12$; the asymptotic behaviour is

$$
\begin{aligned}
& v(\xi) \sim 1-\frac{1}{2 \xi} \ln (2 \pi \xi), \quad \xi \rightarrow \infty, \\
& v(\xi) \sim-\gamma-\frac{1}{2 \xi}, \quad \xi \rightarrow 0,
\end{aligned}
$$

where $\gamma \simeq 0.577$ is the Euler's constant.

In the approximation considered the solution of equation (4) reads

$$
M_{\mathrm{dyn}}=\Lambda \exp \left[-\xi\left(\frac{1}{\tau}-v(\xi)\right)\right] .
$$

To discuss the physical content of this result, we recall that the energy spectrum of relativistic fermions in a constant magnetic field $H$ contains Landau levels

$$
E_{n}\left(p_{z}\right)= \pm \sqrt{\hat{m}^{2}+2|Q H| n+p_{z}^{2}}, \quad n=0,1,2, \ldots
$$

with $p_{z}$ denoting the projection of the 3 -momentum on the $z$-axis, i.e., along the magnetic field. If the fermion mass $\hat{m}$ goes to zero, as in the present case, there is no energy gap between the 
vacuum and the LLL. Thus the integer part of $\xi+1$ gives approximately the number of Landau levels taken into account.

The first term in equation (6) has a clearly defined two-dimensional character with a logarithmic dependence on the cutoff in the corresponding gap equation

$$
1=-\frac{G N_{c}}{2 \pi^{2}}|Q H| \ln \left(\frac{m^{2}}{\Lambda^{2}}\right)
$$

and, therefore, in the condensate (compare with equation (5)). Such a behaviour is associated with the $(1+1)$-dimensional dynamics of the fermion pairing on the energy surface $E_{0}=0$ of the LLL [6]. As long as this term dominates over the second term, $v(\xi)$ in $(6)$, one concludes that the condensate is mainly located on the LLL. Actually this condition is fulfilled nearly everywhere at $\tau<1$. This is obvious for $\xi=1$, since $v(1)=1 / 2+\psi(1)=1 / 2-\gamma \simeq-0.08$ is small compared with $1 / \tau$. For $\xi<1$ we come to the same conclusion after considering the asymptotics of the second term (8). The other formula, (7), can be used to show that the above statement is also true for $\xi>1$, except near the critical region $\tau \rightarrow 1-0$, where $v(\xi)$ dominates; then we must conclude that the condensate spreads over many Landau levels.

In this special case it is possible to find an analytical solution. Indeed, using (7) in equation (6) we obtain

$$
1-\frac{|Q H|}{\Lambda^{2}} \ln \left(\frac{\pi m^{2}}{|Q H|}\right)+\mathcal{O}\left(\frac{m^{2}}{\Lambda^{2}}, \frac{4|Q H|^{2}}{\Lambda^{4}}\right) .
$$

We further suppose that the two following small variables are of the same order

$$
\frac{m^{2}}{\Lambda^{2}} \sim\left(\frac{|Q H|}{\Lambda^{2}}\right)^{2} \sim \epsilon
$$

Then it follows immediately that the term with the logarithm is of order $\sqrt{\epsilon} \ln \sqrt{\epsilon}$ and goes to zero, when $\epsilon \rightarrow 0$. Thus, the gap equation

$$
1-\frac{1}{\tau}=\frac{|Q H|}{\Lambda^{2}} \ln \left(\frac{\pi m^{2}}{|Q H|}\right)+\mathcal{O}(\epsilon)
$$

is valid only in the region near the critical value $\tau \rightarrow 1-0$. The closer $\tau$ to 1 , the smaller is $\epsilon$; Landau levels approach a continuum distribution, and a condensate occupies many levels. The physical reason for the changes found in the behaviour of the condensate is the strength of the four-fermion interaction which becomes essentially important here. The corresponding solution is

$$
M_{\mathrm{dyn}}^{2}=\frac{|Q H|}{\pi} \exp \left[-\frac{\Lambda^{2}}{|Q H|}\left(\frac{1}{\tau}-1\right)\right] .
$$

The near-critical regime which we find here differs from the result of paper [6]. In our case the dynamics of the fermion pairing is essentially a $(3+1)$-dimensional one with a quadratic dependence on the cutoff, to be compared with the previous case in equation (9), where we had a $(1+1)$-dimensional behaviour with only a logarithmic dependence on the cutoff.

\subsection{The extended multi-fermion system in a constant magnetic field}

Now we consider the three flavour case with $\kappa, g_{1}, g_{2} \neq 0$. In the simplest case with the octet flavour symmetry, where current quarks have equal masses $\hat{m}_{u}=\hat{m}_{d}=\hat{m}_{s}$, which we set again zero, the system (2) reduces to a cubic equation with respect to $h \equiv h_{u}=h_{d}=h_{s}$

$$
h^{3}+\frac{\kappa}{12 \lambda} h^{2}+\frac{4 G}{3 \lambda} h+\frac{4 m}{3 \lambda}=0
$$




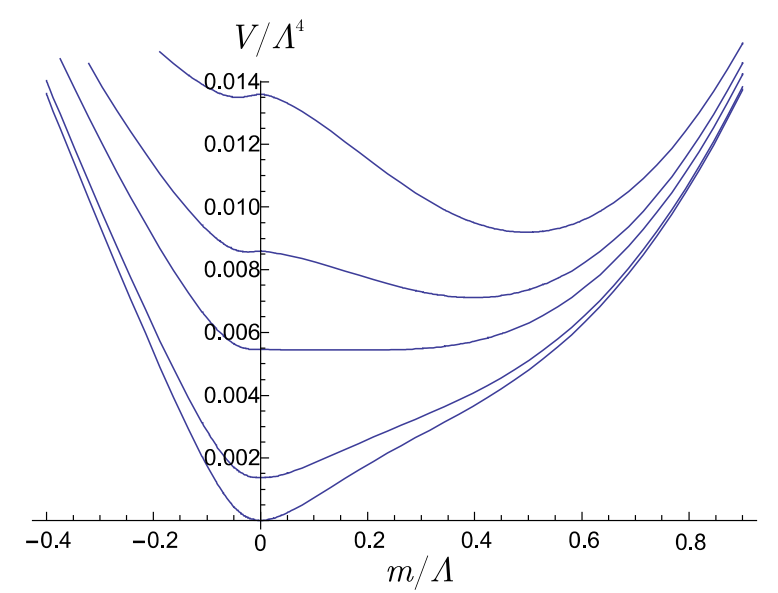

Figure 2. The effective potential $V \Lambda^{-4}$ (in dimensionless units) for the parameters $G \Lambda^{2}=3$, $\kappa \Lambda^{5}=-800, \lambda \Lambda^{8}=1.667 \cdot 10^{3}$. From bottom to top the curves correspond to $|Q H| \Lambda^{-2}=0.05$; $0.1 ; 0.2275 ; 0.3 ; 0.4$.

with $\lambda=g_{1}+(2 / 3) g_{2}$. Making the replacement $h=\bar{h}-\kappa /(36 \lambda)$, one obtains from (10)

$$
\bar{h}^{3}+t \bar{h}=b,
$$

where

$$
t=\frac{4}{3}\left[\frac{G}{\lambda}-\left(\frac{\kappa}{24 \lambda}\right)^{2}\right], \quad b=\frac{\kappa}{27 \lambda}\left[\frac{G}{\lambda}-\frac{2}{3}\left(\frac{\kappa}{24 \lambda}\right)^{2}\right]-\frac{4 m}{3 \lambda} .
$$

This cubic equation has one real root, if $t>0$, i.e.,

$$
\frac{G}{\lambda}>\left(\frac{\kappa}{24 \lambda}\right)^{2}
$$

which is a particular case of the inequalities (3). Assuming that the couplings fulfill condition (12), we find (at any given value of $b$ ) the real solution of equation (11)

$$
\bar{h}(m)=\left(\frac{b}{2}+\sqrt{D}\right)^{\frac{1}{3}}+\left(\frac{b}{2}-\sqrt{D}\right)^{\frac{1}{3}}, \quad D=\left(\frac{t}{3}\right)^{3}+\left(\frac{b}{2}\right)^{2},
$$

with the important property $\bar{h}(0)=\kappa / 36 \lambda$ which ensures that the trivial solution of the gap equation, $m=0$, exists (see [19] for more details about this part of the potential).

In Fig. 2 the effective potential is shown for the parameters $G \Lambda^{2}=3, \kappa \Lambda^{5}=-800, \lambda \Lambda^{8}=$ $1.67 \cdot 10^{3}$. The catalyzed minima close to the origin at small values of the magnetic field get washed out with increasing strength of $H$ giving way to a second minimum at larger values of $M_{\mathrm{dyn}}$. For the lower values of $H$, the catalyzed minimum is so close to the origin that one can not discern it from the trivial maximum which always occurs at the origin (see also discussion around equation (14)). The catalysis of dynamical symmetry breaking can again most easily be seen by considering the non trivial solutions of the corresponding gap equation, to which we turn now.

In a constant magnetic field the gap equation is determined by

$$
-\frac{2 \pi^{2} h(m)}{\Lambda^{2} N_{c} m}=f\left(m^{2} ; \Lambda,|Q H|\right) .
$$

Comparing this result with equation (4), one sees that only the l.h.s. is changed. The sixand eight-quark interactions have modified it in such a way that now we get a function $h(\mathrm{~m}) / \mathrm{m}$ 


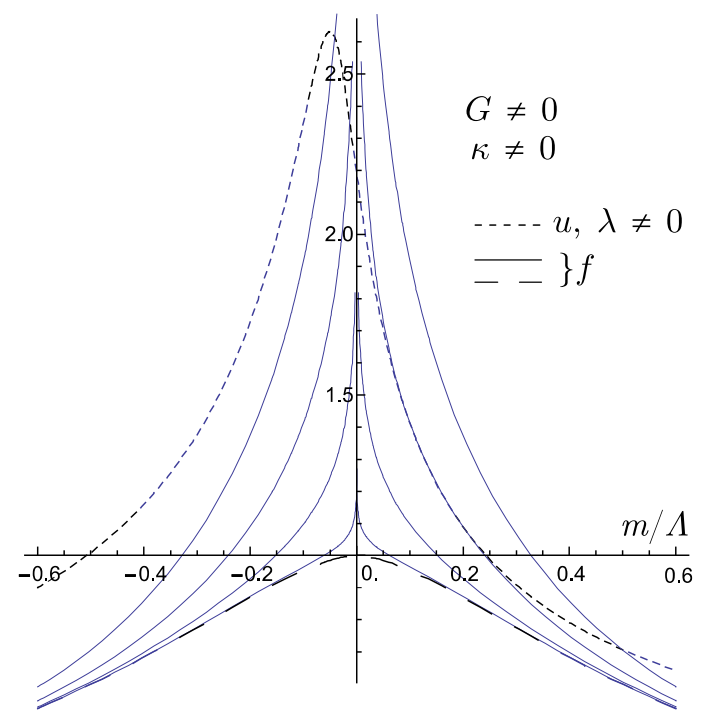

Figure 3. The l.h.s. (short dashed line, label $u$ ) and r.h.s. (label $f$ ) of the gap equation (all in dimensionless units) with the parameter set $G, \kappa, \lambda$ of Fig. 2. From bottom to top the r.h.s. curves have $|Q H| \Lambda^{-2}=0$ (long dashed curve), $|Q H| \Lambda^{-2}=0.02 ; 0.1 ; 0.2275 ; 0.4$ (solid curves). The intersecting solutions $M_{\mathrm{dyn}}$ are displayed in Fig. 4.

instead of the former constant term involving only the coupling of four-quark interactions, $-1 / G$. The l.h.s. of (13), abbreviated by $u$ in Fig. 3, has now a bell-shaped form (short-dashed line), to be compared with the horizontal line of Fig. 1. Since $h(m) / m=-1 / G+\mathcal{O}(m)$, the bell-shaped curve crosses the ordinate axis at the same point as the former straight line (for the same value of $\left.G \Lambda^{2}\right)$. The r.h.s. is again represented by the long-dashed curve ( $H=0$ case) and by the full lines for the finite $H$ cases. As mentioned before these are not altered by the couplings $G, \kappa, \lambda$. The intersection points of the l.h.s. with the r.h.s. curves yield the nontrivial solutions of the gap equation: either one or three solutions can be found for $m>0$. For the parameter set considered the region of coexisting solutions is very narrow, see Fig. 4. If equation (13) has no non-trivial solutions at $H=0$ (the present case) we say that the set of couplings $G, \kappa, \lambda$ is subcritical. It is said to be overcritical in the opposite case. Note that the overcritical set may contain $G<G_{\text {crit }}$ (a parameter set for this case can be found in [22]; there the coexistence of solutions fills a larger interval in $H$, and starts at $H=0$ ).

The trivial solution, $m=0$, corresponds to the point where the potential, $V(m)$, reaches its local maximum. The second derivative

$$
\lim _{m \rightarrow 0} \frac{d^{2} V(m)}{d m^{2}}=\lim _{m \rightarrow 0} \frac{N_{c}|Q H|}{2 \pi^{2}} \ln \frac{|m| \Lambda}{2|Q H|}=-\infty
$$

is negative here. This is the general mathematical reason for the phenomena known as magnetic catalysis of dynamical flavour symmetry breaking. The logarithmically divergent negative result ensures that this phase transition always takes place, if $H \neq 0$. This transition does not depend on the details related with the multi-quark dynamics, i.e., the result is true even for free fermions in a constant magnetic field.

What is really dependent on the multi-quark dynamics is the local minima structure of the theory. Let us recall that in the theory with just four-fermion interactions the effective potential has only one minimum at $m>0$, and this property does not depend on the strength of the field $H$. We have demonstrated this in Fig. 1. In the theory with four-, six-, and eight-quark interactions one can find either one or two local minima at $m>0$. The result depends on the strength of the magnetic field $H$, and couplings $G, \kappa, \lambda$. This is illustrated in Figs. 2-4. Namely, the upper full curve (r.h.s. of equation (13) for $|Q H| \Lambda^{-2}=0.4$ ) has only one intersection point 


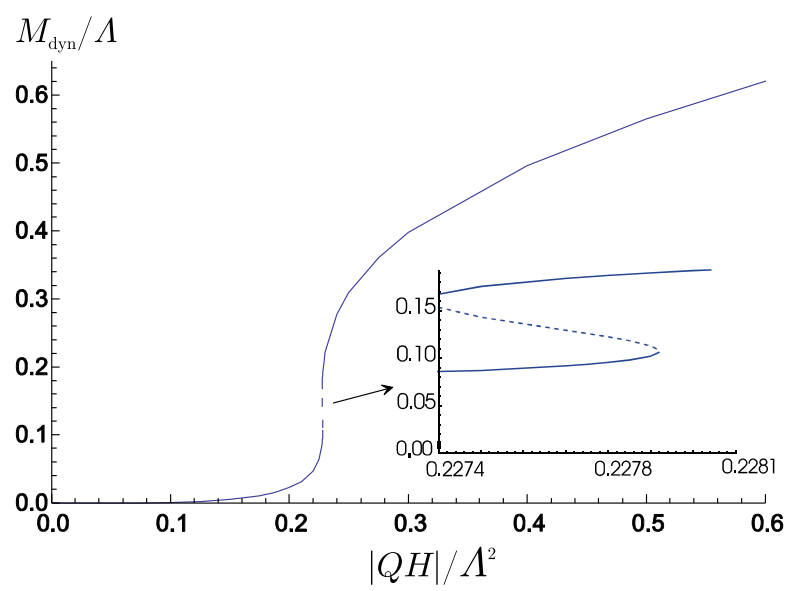

Figure 4. The non-trivial solutions $M_{\mathrm{dyn}} / \Lambda$ of the gap equation, as functions of $|Q H| \Lambda^{-2}$, for the parameter set $G, \kappa, \lambda$ of Figs. 2 and 3. For $0<|Q H| \Lambda^{-2}<0.2274, M_{\text {dyn }}$ corresponds to the catalysis phase. Then a rapid transition leads the system to the larger $M_{\text {dyn }}$ solution induced by the higher order multi-quark forces. A coexistence of both kinds of solutions (at the interrupted line) happens in a very narrow window of $|Q H| \Lambda^{-2}$ values (see box insertion: short dashed line are maxima, solid lines minima). All quantities are dimensionless.

with the bell-shaped curve $u$ (l.h.s. of equation (13) for $G \Lambda^{2}=3, \kappa \Lambda^{5}=-800, \lambda \Lambda^{8}=1667$.), as seen in Fig. 3. This point corresponds to a single vacuum state of the theory at a large $M_{\text {dyn }}$. As opposed to this the curve with $|Q H| \Lambda^{-2}=0.2275$ has three intersections with the same curve $u$; these intersections, taken in order, correspond to a local minimum, a local maximum and a further local minimum of the potential (see also Fig. 4). Reducing further the field strength one obtains again only one solution, at considerable smaller values of $M_{\mathrm{dyn}}$, corresponding to catalysis.

Therefore the first minimum catalyzed by a constant magnetic field is then smoothed out with increasing $H$. It ceases to exist at some critical value of $|Q H| \Lambda^{-2}$, from which on only the large $M_{\text {dyn }}$ solution survives, as shown in Fig. 4, for the parameter set of Figs. 2, 3. This process is accompanied by a sharp increase in depth of the effective potential at the second minimum (see Fig. 2). It is clear that the only way to wash out the first minimum (due to equation (14)), is by lowering the barrier between this state and the second minimum. We also observe that the second minimum is unremovable, because the asymptotic behaviour of the functions in equation (13) is such that the l.h.s. dominates over the r.h.s. at large $m / \Lambda$. Let us stress that this scenario will always be possible if at $H=0$ the six- and eight-quark interactions induce dynamical symmetry breaking in the subcritical regime $G<G_{\text {crit }}$ of fourquark interactions (the case presented in [22]). If the system remains subcritical also for the higher-order interactions, as in the example shown here, the regime of coexistence of more than one minimum of the gap equation at finite $H$ shrinks eventually to zero by lowering further the eight-quark interaction strength $\lambda$ (keeping the remaining parameters fixed). Then one can still observe a crossover transition from small to large $M_{\text {dyn }}$ solutions. For example by changing the r.h.s. of the stability condition $G-\left(\frac{\kappa}{24}\right)^{2} \frac{1}{\lambda}>0$ from 2.33 (parameter set shown in Figs. 2-4) to 1., one obtains qualitatively the same picture as in Fig. 4 (of course without coexistence region) but a smoother transition.

\section{Concluding remarks}

The effects of interactions among fermions on catalysis of dynamical chiral symmetry breaking by a constant magnetic field have been considered. A generalized NJL model with $U(3)$ flavor symmetry containing up to eight-quark interactions has been at the basis of this study, and the 
motivation for the use of such a Lagrangian was briefly reviewed. A critical survey of the nontrivial solutions of the gap equations has been obtained and compared to the known solutions of the conventional four-quark interaction NJL model.

We have discussed two cases: (i) all coupling strengths are subcritical, (ii) the addition of six and eight quark interactions transform the original subcritical $G<G_{\text {crit }}$ four-quark system into an overcritical one, inducing the formation of a large condensate.

In both cases we have that in the region $m^{2} / \Lambda^{2} \ll 1$ the four-fermion interaction dominates the behaviour of the system. Since their coupling strength is small, $G<G_{\text {crit }}$, the massless fermions behave like almost free particles moving in a weak external magnetic field, with access to a large number of Landau levels, $\xi \gg 1$. This field catalyzes the process of fermion-antifermion pairing on the energy surface $E_{0}=0$ of the LLL. The first minimum localized at $m^{2} / \Lambda^{2} \ll 1$ is exactly formed by such an (1+1)-dimensional condensate. If six- and eight-fermion forces would not act on the system, this ground state would be stable: our formulae as well as the result of paper [6] show clearly that a slow increase of the strength $H$ does not wash out the condensate from the energy surface $E_{0}=0$ of the LLL. However, when the six- and eight-fermion interactions are present, a slowly increasing magnetic field destroys finally this ground state. In case (ii) this always happens; in case (i) the transition can either be sharp as in (ii) or of a crossover type, depending on the interaction strengths. The new condensate has a $(3+1)$-dimensional structure similar in every respect to the standard NJL case with broken chiral symmetry at $H=0$, i.e., when the condensate spreads over many single fermion states. This is because the increasing magnetic field enlarges the dynamical fermion mass, and scales of order $m / \Lambda \sim 1$ become relevant. At these scales the 't Hooft and eight-quark interactions push the system to a new regime, where the fermions are not anymore free-like particles: they interact strongly with each other and this interaction changes essentially the fermionic spectrum and the structure of the ground state with all the above mentioned consequences.

Thus we have obtained not only a correct description of the well-known physics related with the LLL, but have found also a clear signature for the possibly important role played by 't Hooft and eight-quark interactions. Namely, in the presence of these interactions the magnetic field can change the condensation zone from the zero-energy surface of the LLL to a wide region spread over many Landau levels and vice versa. One can expect that hard gamma emissions accompany this process.

The value of the characteristic scale $\Lambda$ has not been specified yet. We assume that this value is determined by the problem under consideration. Its choice can also be motivated by the number of Landau levels to be considered. The characteristic scale of the magnetic fields which can induce such a transition is of the order $H=7 \cdot 10^{13} \Lambda^{2}$ Gauss $/ \mathrm{MeV}^{2} \mathrm{~s}$, and actually depend on the cutoff involved in the problem. For instance, in hadronic matter it is probably reasonable to assume that $\Lambda \simeq 800 \mathrm{MeV}$, leading to $H=4.5 \cdot 10^{19} \mathrm{G}$.

A potentially interesting area where this effect may find applications are studies of compact stellar objects in presence of strong magnetic fields, in particular the young neutron stars, magnetars [34]. The surface magnetic fields are observed to be $\geq 10^{15} \mathrm{G}$, but actually they can be even much higher at the core region. Another area is connected with the electroweak phase transition in the early Universe [35], where the strength of magnetic fields can reach $H \sim 10^{24}$ G. Even giving conservative limitations on the primordial magnetic field of present epoch value of $10^{-9} \mathrm{G}$, based on primordial nuclear synthesis calculations [36], it seems clear that the magnetic field reached the transition regime discussed in this paper in the past. A very rough estimate based on the relation $H \propto T_{\nu}^{2}\left(T_{\nu}\right.$ being the neutrino temperature), gives a magnetic field strength at baryogenesis of at least $10^{17} \mathrm{G}$, of $10^{21} \mathrm{G}$ at the epoch of electroweak breaking and of $10^{45} \mathrm{G}$ at the GUT scale. The coupling to those strong magnetic fields will have influence on those processes. However to incorporate the findings of the present work into those mechanisms is beyond the scope of the present article. 


\section{Acknowledgements}

B. Hiller is very grateful to the organizers for the kind invitation and hospitality and for creating the conditions for a very interesting conference. This work has been supported in part by grants provided by Fundação para a Ciência e a Tecnologia, POCI 2010 and FEDER, POCI/FP/63930/2005, POCI/FP/81926/2007. This research is part of the EU integrated infrastructure initiative Hadron Physics project under contract No.RII3-CT-2004-506078.

\section{References}

[1] Jackiw R., Fractional charges and zero modes for planar systems in a magnetic field, Phys. Rev. D 29 (1984), 2375-2377.

Barducci A., Casalbuoni R., Lusanna L., Supersymmetries and the pseudoclassical relativistic electron, Nuovo Cimento A 35 (1976), 377-399.

[2] Klevansky S.P., Lemmer R.H., Chiral symmetry restoration in the Nambu-Jona-Lasinio model with a constant electromagnetic field, Phys. Rev. D 39 (1989), 3478-3489.

[3] Klimenko K.G., Three-dimensional Gross-Neveu model in an external magnetic field, Theoret. and Math. Phys. 89 (1992), 1161-1168.

Vshivtsev A.S., Klimenko K.G., Magnitsky B.V., Vacuum structure of the $(\psi-\bar{\psi})^{2}$ model, accounting for the magnetic field and chemical potential, Theoret. and Math. Phys. 106 (1996), 319-327.

[4] Krive I.V., Naftulin S.A., Electrodynamics of systems with dynamical generation of mass in (2+1)dimensional space-time, Sov. J. Nuclear Phys. 54 (1991), 897-902.

Krive I.V., Naftulin S.A., Dynamical symmetry breaking and phase transitions in a three-dimensional GrossNeveu model in a strong magnetic field, Phys. Rev. D 46 (1992), 2737-2740.

[5] Gusynin V.P., Miransky V.A., Shovkovy I.A., Catalysis of dynamical flavor symmetry breaking by a magnetic field in (2+1)-dimensions, Phys. Rev. Lett. 73 (1994), 3499-3502, hep-ph/9405262.

[6] Gusynin V.P., Miransky V.A., Shovkovy I.A., Dimensional reduction and dynamical chiral symmetry breaking by a magnetic field in (3+1)-dimensions, Phys. Lett. B 349 (1995), 477-483, hep-ph/9412257.

Gusynin V.P., Miransky V.A., Shovkovy I.A., Dynamical flavor symmetry breaking by a magnetic field in (2+1)-dimensions, Phys. Rev. D 52 (1995), 4718-4735, hep-th/9407168.

[7] Gusynin V.P., Miransky V.A., Shovkovy I.A., Dimensional reduction and catalysis of dynamical symmetry breaking by a magnetic field, Nuclear Phys. B 462 (1996), 249-290, hep-ph/9509320.

[8] Bardeen J., Cooper L.N., Schrieffer J.R., Theory of superconductivity, Phys. Rev. 108 (1957), 1175-1204.

[9] Ragazzon R., The Nambu-Jona-Lasinio in simple configurations of strong and non-homogeneous magnetic configuartions, Phys. Lett. B 334 (1994), 427-430.

Dunne G., Hall T., Inhomogeneous condensates in planar QED, Phys. Rev. D 53 (1996), 2220-2226, hep-th/9511192.

[10] Ragazzon R., Nambu-Jona-Lasinio model in a magnetic field with variable direction, Phys. Rev. D 59 (1999), 065006, 5 pages.

[11] Nambu Y., Jona-Lasinio G., Dynamical model of elementary particles based on an analogy with superconductivity. I, Phys. Rev. 122 (1961), 345-358.

Nambu Y., Jona-Lasinio G., Dynamical model of elementary particles based on an analogy with superconductivity. II, Phys. Rev. 124 (1961), 246-254.

Vaks V.G., Larkin A.I., On the application of the methods of superconductivity theory to the problem of the masses of elementary particles, Zh. Eksp. Teor. Fiz. 40 (1961), 282-285 (English transl.: Sov. Phys. JETP 13 (1961), 192-193).

Arbuzov B.A., Tavkhelidze A.N., Faustov R.N., On the fermion mass in the $\gamma_{5}$-invariant model of the quantum field theory, Dokl. Akad. Nauk. SSSR 139 (1961), 345-347 (English transl.: Sov. Phys. Dokl. 6 (1962), 598-600).

[12] Osipov A.A., Hiller B., da Providência J., Multi-quark interactions with a globally stable vacuum, Phys. Lett. B 634 (2006), 48-54, hep-ph/0508058.

[13] 't Hooft G., Computation of the quantum effects due to a four-dimensional pseudoparticle, Phys. Rev. D 14 (1976), 3432-3450, Erratum, Phys. Rev. D 18 (1978), 2199.

[14] Osipov A.A., Hiller B., Bernard V., Blin A.H., Aspects of $U_{A}(1)$ breaking in the Nambu and Jona-Lasinio model, Ann. Phys. 321 (2006), 2504-2534, hep-ph/0507226. 
[15] Hiller B., Osipov A.A., Bernard V., Blin A.H., Functional integral approaches to the bosonization of effective multi-quark interactions with $U_{A}(1)$ breaking, SIGMA 2 (2006), 026, 18 pages, hep-ph/0602165.

[16] Zwanziger D., Private communication.

[17] Simonov Yu.A., Chiral Lagrangian with confinement from the QCD Lagrangian, Phys. Rev. D 65 (2002), 094018, 10 pages, hep-ph/0201170.

[18] Bali G.S., QCD forces and heavy quark bound states, Phys. Rep. 343 (2001), 1-136, hep-ph/0001312.

[19] Osipov A.A., Hiller B., Blin A.H., da Providência J., Effects of eight-quark interactions on the hadronic vacuum and mass spectra of light mesons, Ann. Phys. 332 (2007), 2021-2054, hep-ph/0607066.

[20] Osipov A.A., Hiller B., Moreira J., Blin A.H., da Providência J., Lowering the critical temperature with eight-quark interactions, Phys. Lett. B 646 (2007), 91-94, hep-ph/0612082.

Osipov A.A., Hiller B., Moreira J., Blin A.H., OZI violating eight-quark interactions as a thermometer for chiral transitions, Phys. Lett. B 659 (2008), 270-274, arXiv:0709.3507.

[21] Kashiwa K., Kouno H., Sakaguchi T., Matsuzaki M., Yahiro M., Chiral phase transition in an extended NJL model with higher-order multi-quark interactions, Phys. Lett. B 647 (2007), 446-451, nucl-th/0608078.

Kashiwa K., Kouno H., Matsuzaki M., Yahiro M., Critical endpoint in the Polyakov-loop extended NJL model, arXiv:0710.2180.

[22] Osipov A.A., Hiller B., Blin A.H., da Providência J., Dynamical chiral symmetry breaking by a magnetic field and multi-quark interactions, Phys. Lett. B 650 (2007), 262-267, hep-ph/0701090.

[23] Okubo S., Phi meson and unitary symmetry model, Phys. Lett. B 5 (1963), 165-168.

Zweig G., An $S U(3)$ model for strong interaction symmetry and its breaking. 2, CERN Report No. 8419/TH412, 1964.

Iizuka I., A systematics and phenomenology of meson family, Progr. Theoret. Phys. Suppl. 37-38 (1966), 21-34.

[24] Eguchi T., New approach to collective phenomena in superconductivity models, Phys. Rev. D 14 (1976), $2755-2763$.

Kikkawa K., Quantum corrections in superconductor models, Progr. Theoret. Phys. 56 (1976), 947-955.

[25] Volkov M.K., Ebert D., Four-quark interactions as a common dynamical basis of the $\sigma$ model and the vector dominance model, Sov. J. Nuclear Phys. 36 (1982), 736-742.

Ebert D., Volkov M.K., Composite meson model with vector dominance based on $U(2)$ invariant four quark interactions, Z. Phys. C 16 (1983), 205-210.

[26] Dhar A., Wadia S., The Nambu-Jona-Lasinio model: an effective Lagrangian for quantum chromodynamics at intermediate length scales, Phys. Rev. Lett. 52 (1984), 959-962.

Dhar A., Shankar R., Wadia S., Nambu-Jona-Lasinio type effective Lagrangian: anomalies and nonlinear Lagrangian of low-energy, large-N QCD, Phys. Rev. D 31 (1985), 3256-3267.

Ebert D., Reinhardt H., Effective chiral hadron Lagrangian with anomalies and Skyrme terms from quark flavour dynamics, Nuclear Phys. B 271 (1986), 188-226.

Schüren C., Arriola E.R., Goeke K., Explicit chiral symmetry breaking in the Nambu-Jona-Lasinio model, Nuclear Phys. A $\mathbf{5 4 7}$ (1992), 612-632.

Bijnens J., Bruno C., de Rafael E., Nambu-Jona-Lasinio like models and the low energy effective action of QCD, Nuclear Phys. B 390 (1993), 501-541, hep-ph/9206236.

Bernard V., Osipov A.A., Meißner U.-G., The momentum-space bosonization of the Nambu-Jona-Lasinio model with vector and axial-vector mesons Phys. Lett. B 324 (1994), 201-208, hep-ph/9312203.

Bernard V., Blin A.H., Hiller B., Ivanov Yu.P., Osipov A.A., Meißner U.-G., Pion observables in the extended NJL model with vector and axial-vector mesons Ann. Phys. 249 (1996), 499-531, hep-ph/9506309.

Osipov A.A., Hiller B., Effective chiral meson Lagrangian for the extended Nambu-Jona-Lasinio model, Phys. Rev. D 62 (2000), 114013, 10 pages, hep-ph/0007102.

Osipov A.A., Sampaio M., Hiller B., Implications of a new effective chiral meson Lagrangian, Nuclear Phys. A 703 (2000), 378-392, hep-ph/0110285.

Osipov A.A., Hiller B., Generalized proper time approach for the case of broken isospin symmetry, Phys. Rev. D 63 (2001), 094009, 10 pages, hep-ph/0012294.

[27] Bernard V., Jaffe R.L., Meißner U.-G., Flavor mixing via dynamical chiral symmetry breaking, Phys. Lett. B 198 (1987), 92-98.

Bernard V., Jaffe R.L., Meißner U.-G., Strangeness mixing and quenching in the Nambu-Jona-Lasinio model, Nuclear Phys. B 308 (1988), 753-790.

[28] Reinhardt H., Alkofer R., Instanton induced flavour mixing in mesons, Phys. Lett. B 207 (1988), $482-488$. 
[29] Klimt S, Lutz M., Vogl U., Weise W., Generalized SU(3) Nambu-Jona-Lasinio model. Part 1. Mesonic modes, Nuclear Phys A 516 (1990), 429-468.

Vogl U., Lutz M., Klimt S., Weise W., Generalized SU(3) Nambu-Jona-Lasinio model. Part 2. From current to constituent quarks, Nuclear Phys A 516 (1990), 469-495.

[30] Bernard V., Blin A.H., Hiller B., Meißner U.-G., Ruivo M.C., Strong and radiative meson decays in a generalized Nambu-Jona-Lasinio model, Phys. Lett. B 305 (1993), 163-167, hep-ph/9302245.

Dmitrasinovic V., $U_{A}(1)$ symmetry breaking, scalar mesons and the nucleon spin problem in an effective chiral field theory, Nuclear Phys. A 686 (2001), 379-392, hep-ph/0010047.

[31] Klevansky S.P., The Nambu-Jona-Lasinio model of quantum chromodynamics, Rev. Modern Phys. 64 (1992), 649-708.

Hatsuda T., Kunihiro T., QCD Phenomenology based on a chiral effective Lagrangian, Phys. Rep. 247 (1994), 221-367, hep-ph/9401310.

[32] Schwinger J., On gauge invariance and vacuum polarization, Phys. Rev. 82 (1951), 664-679.

[33] Bateman H., Erdelyi A., Higher transcendental functions, Mc Graw-Hill Book Company, Inc., 1953.

[34] Duncan R.C., Thompson C., Formation of very strongly magnetized neutron stars - implications for gammaray bursts, Astrophys. J. Lett. 392 (1992), L9-L13.

Thompson C., Duncan R.C., Neutron star dynamos and the origins of pulsar magnetism, Astrophys. J. 408 (1993), 194-203.

Thompson C., Duncan R.C., The soft gamma repeaters as very strongly magnetized neutron stars. II. Quiescent neutrino, X-ray, and Alfven wave emission, Astrophys. J. 473 (1996), 322-342.

Kouveliotou C. et al., An X-ray pulsar with a superstrong magnetic field in the soft gamma-ray repeater SGR 1806-20, Nature 393 (1998), 235-237.

Kouveliotou C. et al., Discovery of a magnetar associated with the soft gamma repeater SGR 1900+14, Astrophys. J. 510 (1999), L115-L118, astro-ph/9809140.

Woods P.M. et al., Discovery of a new soft gamma repeater, SGR 1627-41, Astrophys. J. Lett. 519 (1999), L139-L142, astro-ph/9903267.

[35] Vachaspati T., Magnetic fields from cosmological phase transitions, Phys. Lett. B 265 (1991), $258-261$. Olesen P., On the possible creation of a background W condensate in the electroweak phase transition, Phys. Lett. B 281 (1992), 300-302.

[36] Yamazaki D.G., Ichiki K., Kajino T., Mathews G.J., Magnetic field constrained from CMB anisotropies,and its generation and evolution before, during and after the BBN, in Proceedings of the International Symposium on Nuclear Astrophysics "Nuclei in the Cosmos-IX" (June 25-30, 2006, CERN, Geneva), Proceedings of Science, PoS(NIC-IX), 2006, 194-199, astro-ph/0610234.

Kernan P.J., Starkman G.D., Vachaspati T., Big bang nucleosynthesis constraints on primordial magnetic fields, Phys. Rev. D 54 (1996), 7207-7214, astro-ph/9509126.

Cheng B., Olinto A.V., Schramm D.N., Truran J.W., Constraints on the strength of primordial magnetic fields from big bang nucleosynthesis revisited, Phys.Rev. D 54 (1996), 4714-4718, astro-ph/9606163. 